\title{
A Database Tool for Raising Awareness on the Life Cycle Environmental Impacts of Household Packaging Waste
}

\author{
Konstadinos Abeliotis, Fotios Zachos, and Katia Lasaridi \\ School of Environment, Geography and Applied Economics, Harokopio University, El. Venizelou 70, 17671 Athens, Greece \\ Correspondence should be addressed to Konstadinos Abeliotis; kabeli@hua.gr
}

Received 29 October 2013; Revised 24 December 2013; Accepted 25 December 2013; Published 3 February 2014

Academic Editor: Wen-Cheng Liu

Copyright ( 2014 Konstadinos Abeliotis et al. This is an open access article distributed under the Creative Commons Attribution License, which permits unrestricted use, distribution, and reproduction in any medium, provided the original work is properly cited.

Packaging is a key stage of the life cycle of a product regarding its environmental impact. Also, the contribution of the households in the success of packaging recycling programs is well documented. The aim of this paper is the presentation of the development of a computer-aided tool that assists consumers in minimising their everyday environmental impact via the environmental assessment of the products' packaging coupled with the recycling behaviour of the consumer. The tool has been developed in Microsoft Access and consists of a user-friendly interface and four databases which contain data on the packaging materials of consumer products and their weights alongside with data for the recycling rates of various waste packaging materials in Greece. The user inputs the number of packages per product that he/she bought followed by his/her recycling habits. The tool then calculates the environmental impacts of his/her choices (both consumer and recycling). The key advantage of the developed computer tool is its simplicity, both conceptual and operable. On the other hand, its main drawback is that impact assessment data are based on Dutch conditions. Overall, the implications of the developed computer-aided tool for informing and raising consumer awareness on packaging waste recycling are enormous.

\section{Introduction}

Packaging is an indispensable part of every consumer product [1]. Packaging offers preservation of product from contamination, product information and identification, and convenient transport and distribution. Packaging waste constitutes one of the major streams in municipal solid waste. It is estimated that at present there is about 10 million tons of solid waste generated annually in the world, of which packaging waste accounts for one-third [2]. Packaging has been the subject of intense public debate, as today's products are offered in a wide range of packaging alternatives made from different materials and in different sizes [3] and because the packaging waste stream is among the most rapidly growing components of municipal solid waste since at least the 1970s [4]. Life cycle assessment [5] is a holistic methodology, utilized routinely for the environmental impact assessment of packaging materials $[6,7]$ since the $1990 \mathrm{~s}$, following the pioneering work by Hocking [8]. Environmental impact assessment is based on the data acquired during the life cycle inventory phase of each packaging material. The utilisation of such databases for the compilation of life cycle data is well established. Among them, the ecoinvent database is the most recognisable [9].

Proper management of packaging waste is required in order to improve the overall environmental efficiency of any product. In this framework, extended producer responsibility (ERP) is being propagated as a new paradigm in packaging waste management by engaging both producers and consumers [10]. Based on its importance, management of packaging waste is among the first issues tackled by the EPR schemes worldwide. The European Union has adopted the key pillars of EPR and implemented them, through various directives, in corresponding waste streams [9]. Producers may fulfill their responsibility either in an individual or a collective way. If they choose the first, they bear responsibility only for the products that they put on market. However, bearing the responsibility may be proved extremely costly. Instead, the producers can join together and form collective compliance schemes (or collective take-back systems), in order to be in line with EPR requirements. The role of 
TABLE 1: Packaging material recycled in Greece (2008-2010) [12].

\begin{tabular}{|c|c|c|c|c|c|c|c|c|c|}
\hline \multirow[b]{2}{*}{ Packaging material } & \multicolumn{3}{|c|}{2008} & \multicolumn{3}{|c|}{2009} & \multicolumn{3}{|c|}{2010} \\
\hline & $\begin{array}{l}\text { Produced } \\
\quad(\mathrm{tn})\end{array}$ & $\begin{array}{c}\text { Recycled } \\
\text { (tn) }\end{array}$ & $\%$ recycled & $\begin{array}{l}\text { Produced } \\
(\text { tn })\end{array}$ & $\begin{array}{c}\text { Recycled } \\
\text { (tn) }\end{array}$ & $\%$ recycled & $\begin{array}{l}\text { Produced } \\
(\mathrm{tn})\end{array}$ & $\begin{array}{c}\text { Recycled } \\
\text { (tn) }\end{array}$ & $\%$ recycled \\
\hline Glass & 160,000 & 24,000 & 15 & 155,000 & 23,500 & 15.2 & 135,100 & 28,923 & 21.4 \\
\hline Plastic & 240,000 & 28,605 & 11.9 & 237,000 & 63,200 & 26.7 & 221,500 & 66,730 & 30.1 \\
\hline Paper/cardboard & 440,000 & 324,056 & 73.6 & 430,000 & 357,000 & 83 & 392,900 & 369,780 & 94.1 \\
\hline Metals & 145,000 & 63,500 & 43.8 & 131,800 & 63,500 & 48.2 & 127,400 & 53,360 & 41.9 \\
\hline Wood & 65,000 & 20,002 & 30.8 & 54,200 & 20,200 & 37.3 & 50,500 & 26,841 & 53.2 \\
\hline Total & $1,050,000$ & 460,163 & 43.8 & $1,008,00$ & 527,400 & 52.3 & 927,400 & 545,634 & 58.8 \\
\hline
\end{tabular}

the aforementioned schemes, which are known as Producer Responsibility Organisations (PROs), is pivotal in the EPR implementation. Till today, packaging waste, end-of-life vehicles, waste electrical and electronic equipment, and batteries are regulated under EPR schemes all over the European Union [11], while the recent Waste Framework Directive 2008/98/EC introduced in a more explicit way the EPR concept.

The aim of this paper is the presentation of the development of a computer-aided tool which calculates the environmental impact of the packaging waste associated with the everyday supply of consumer products. The tool is built utilising the characteristics of relational databases. The required inputs to the tool are a list of consumer products in addition to the recycling habits of the consumer and the recovery rates achieved by the various packaging recycling systems currently operating in Greece. The tool then, based on the available recycling rates per material and a predefined impact scale, calculates the total impact caused by the packaging waste of the initial list of products.

\section{Packaging Recycling in Greece}

The packaging waste components recycled in Greece are plastic, glass, aluminum, paper, paperboard, tin, and wood via either collective or individual PRO schemes. The PRO with the most widespread geographical coverage is that of the "blue" bins, since it is a collective one. During 2011, the coverage of the Greek population by the PRO system for packaging waste reached 79\% (based on the population data of the 2001 census). There is also an individual PRO operated by a major food retailer and another collective one aiming specifically at the plastic packages of lubricants. Once collected, the content of the "blue" bins is transferred to recycling centres, where the recyclables are separated into material streams. However, there is a quite high percentage of the "blue" bins content (ranging from 17 to $45 \%$ for 2010 ) that ends up in the landfill, due to low material quality.

The amount of each packaging material recycled in Greece from 2008 to 2010 is presented in Table 1 [12]. The quantitative targets for the end of 2011 for packaging waste were set to $55-80 \%$ recycling, by weight. Regarding the recovery rate, the latest updated data, available for 2010, that is, seven years after the introduction of the collective "blue" bins system, indicate that 545,634 tons of packaging waste was
TABLE 2: Recovery rates per material via the "blue bins" collective system [12].

\begin{tabular}{lc}
\hline Material & \% in the blue bin (by weight) \\
\hline Paper (printed) & 42.0 \\
Paper (packaging) & 35.3 \\
Glass & 3.1 \\
Tin & 2.4 \\
Aluminum & 0.9 \\
Plastic & 2.7 \\
PET bottles & 4.1 \\
Polyethylene bottles & 2.8 \\
Polyethylene film & 4.4 \\
Others & 2.3 \\
\hline
\end{tabular}

recycled, a quantity which corresponds to $58.8 \%$ of the total packaging waste [12]. The average $\%$ content per material of the blue bins for 2010 is presented in Table 2 [12].

Regarding the temporal trend of packaging waste recycling, its quantities in 2011 were reduced compared to 2008 by $19 \%$ ( $4 \%$ during $2009,8 \%$ during 2010 , and $7 \%$ during 2011). 194,000 tonnes of packaging waste was recycled in 2011, along with 274,000 tonnes of recyclable materials (including printed paper). During the same year, the per capita packaging waste recovery index was reduced by approximately $11 \%$, for the first time since 2004 [12]. This is not only the result of both the financial crisis that Greece is facing but also it is attributed to the activity of scavengers, which has been increased, also as a result of the general economic and social decline in Greece. However, note that there was a significant increase $(+54 \%)$ in the recovery of glass compared to 2010 as a result of the focused actions for its recovery [12].

\section{The Implementation of the Tool}

The goal of the developed computer-aided tool is the identification of the environmental impact caused by the packaging of everyday items, which subsequently aims at raising environmental consciousness by the consumers. In order to achieve its goal, the tool utilises a set of databases which contain numerical data manipulated by a set of logical predefined criteria. Thus, the developed tool falls within the hybrid DSS approaches. The advantages of databases for increased information sharing and advanced environmental consciousness 
of individuals related to green products are well documented [13]. The computer-aided tool was developed in Microsoft Access 2000, a relational database that comes together with its Data Base Management System (DBMS) that runs in Windows XP operational system on a personal computer. It is a standalone software package that makes possible the overall management of structured information, that is, its storage, selective search, and retrieval.

Microsoft Access includes wizards that easily create the data files and the commands for comparing, retrieving, printing, and presenting the data. It also includes two "query" languages, namely, the Structured Query Language (SQL) and the Query By Example (QBE). It also includes a full programming language which allows for the development of any calculating or logical algorithm. This language was very helpful for the structuring of the impact improvement algorithm of the tool (see Section 3.2). It also includes a simple programming language for the management of macros.

Once the development of the database application is completed, a single file is created, name. $m d b$, which contains all the databases, queries, macros, and so forth. Thus, the tool can be copied and transferred very easily. All of the aforementioned characteristics make Microsoft Access an ideal tool for the development of low-budget computer tools for research purposes. The only prerequisite for running the tool is the availability of a personal computer with Microsoft Access installed. The user interface of the tool was developed in the Greek language and consists of several dialogue screens.

Figure 1 presents the execution algorithm of the tool. The user, via the introductory program menu, can either run the program and calculate the environmental impact or change the default parameters for the various packaging materials. By pressing the "Change parameters" button, the user has three options:

(i) either to introduce new products in the products database or change the packaging characteristics of the existing products or

(ii) modify the default recycling rates per material included in the respective database, or finally

(iii) update the impact assessment default values per material.

If the user chooses to calculate the environmental impact of packaging, there are four major stages in the execution of the program (see Figure 2).

The consumer, at first, inputs the quantities of goods that he/she bought via a dialogue screen that includes a list of all products available in the database (see Figure 3). The function of the program is utilised by the use of a product database which contains packaging information of products that are currently available in the Greek market. The database currently contains packaging information of a hundred products, but the user can extend it by adding new consumer products by just identifying its packaging materials and by weighting them in an ordinary kitchen scale found in every household. In the database, each product record consists of the description of the product, its packaging material(s), and

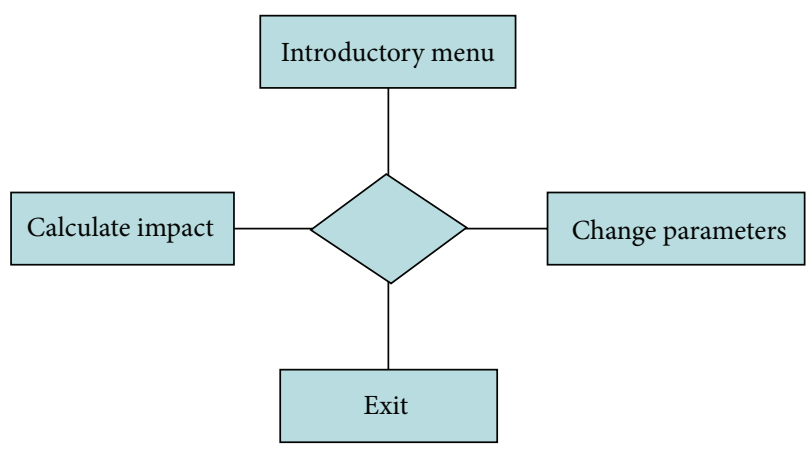

Figure 1: The execution algorithm for the developed tool.

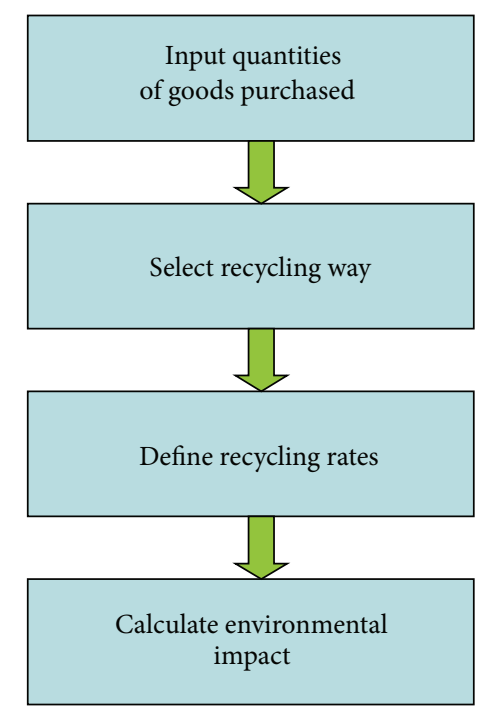

FIGURE 2: Outline of the tool algorithm.

the weight of the packaging material(s). In the upper righthand side of the product definition dialogue screen, there is also a button which once pressed resets all product selection values to zero.

The tool, then, prompts the consumer to identify his/her recycling habits for each one of the materials, that is, glass, aluminum, various plastics (PET, PVC, PP, HDPE, and LDPE), and so forth. The next requirement is for the user to check or alter the available recycling rates for each packaging material. Defaults rates are available for each material. The main underlying assumption here is that different packaging waste collection systems yield different recovery rates, even if they are implemented in the same sociodemographic and geographical setting [14]. Recycling rates per material can be updated based on the information published every year by each PRO in the website of the Hellenic Recycling Agency [12].

The tool then calculates the environmental impact of the consumer goods that were initially selected by the user, its recycling habits, the recycling rates, and the life cycle environmental impact of the packaging material(s). The calculation of the life cycle environmental impact is based on the utilisation of the Eco-indicator 99 [15]. Eco-indicator 99 can 


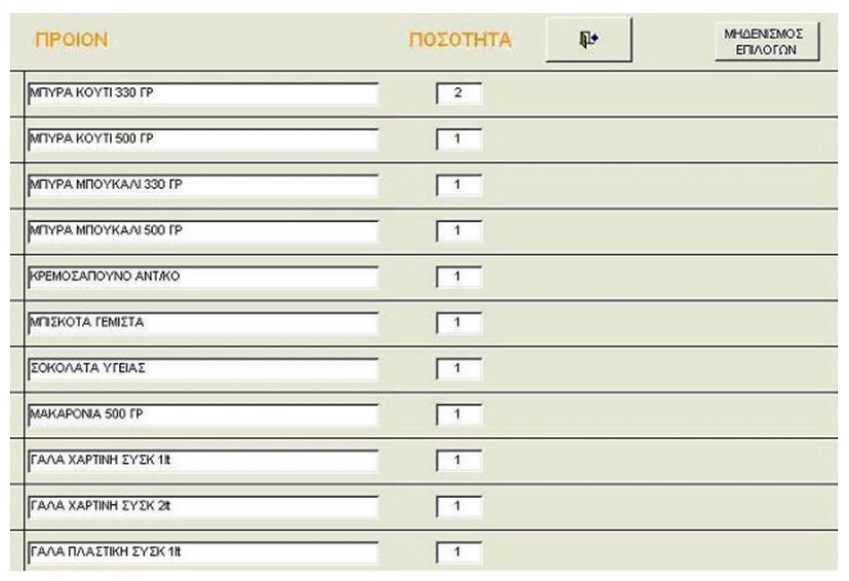

FIGURE 3: Input of consumer products.

be used to calculate standard indicator scores for frequently used materials, such as the packaging materials, during their entire life cycle. It accounts for three principal categories of environmental impacts: human health, ecosystems quality, and resources [15]. The standard Eco-indicator values are dimensionless. The unit of measurement is called the Ecoindicator point, $\mathrm{Pt}$, and is divided into 1000 millipoints (mPts). Practically speaking, the Eco-indicator point is an arbitrary unit, since the kilopoint (=1000 points) is defined by dividing the total environmental load in Europe by the number of its inhabitants [15].

For each material, its production, processing, waste treatment, and recycling, mPts are summed up (see (1)). The total environmental impact is calculated in mPts as follows:

Total impact (mPts)

$$
\begin{aligned}
& =\sum\left[\text { material }_{i} *\left(100-\% \_ \text {recycling_material }{ }_{i}\right)\right. \\
& \text { * material_impact }{ }_{i} \text { ], }
\end{aligned}
$$

where material $_{i}$ is the weight per packaging material expressed in g, \%_recycling_material ${ }_{i}$ is the percentage of recycling per packaging material, and material_impact ${ }_{i}$ is the total environmental impact per packaging material expressed in mPts.

Finally, the consumer can run via the tool an algorithm which suggests a better way of recycling or similar products with lower environmental impact packaging.

3.1. Description of the Databases and Queries. The aforementioned user input is expressed in terms of the following three databases:

(1) ProductsTable: the database which contains all the product-related data;

(2) HowDoYouRecycleTable: the database which contains information on the $\%$ of recycling per packaging material;

(3) TotalMillipointsTable: the database which contains the impact assessment information $(\mathrm{mP} / \mathrm{kg}$ of packaging material).
The database fields included in the ProductsTable are the following:

(i) ID: a numeric variable; each product is assigned a unique identification number;

(ii) Product: a string variable: the full commercial description of the product; it includes common commercial weights of available products;

(iii) Quantity: a numeric variable containing the quantity of packages per product bought by the consumer;

(iv) Rec_pos: a Boolean variable for declaring the possibility of packaging recycling;

(v) Substitute: a numeric variable pointing to a different product identification number in the database which can substitute the current product; substitute products refer to common products available in different packaging and container alternatives (e.g., beer is offered in $500 \mathrm{~mL}$ glass bottles or $330 \mathrm{~mL}$ aluminum cans);

(vi) Material: packaging materials included in the database are glass, aluminum, tin, paper, PET, polyethylene (both high and low density), PVC, and polypropylene;

(vii) WeightperMat: there are as many of those numeric variables as there are packaging materials; each variable contains the respective weight per packaging material; these values are utilized in the calculation of the materials' weights that are recycled;

(viii) TotPackW: a numeric variable that contains the sum of packaging weight per product.

The database fields included in the HowDoYouRecycleTable are the following:

(i) Al_sep: a Boolean variable that is true when the consumer recycles aluminum separately;

(ii) Gl_sep: a Boolean variable that is true when the consumer recycles glass separately;

(iii) PAP_sep: a Boolean variable that is true when the consumer recycles paper separately;

(iv) Blue_bins: a Boolean variable that is true when the consumer recycles all packaging through the blue bins collective system;

(v) No_rec: a Boolean variable that is true when the consumer does not recycle any packaging material at all.

Tables are associated with each one of the aforementioned Boolean variables that include the \% recycling rates per packaging material. Once a variable is selected, the algorithm uses the default recovery values for each one of the packaging materials. The main underlying assumption is that the recovery rates for the selective recycling of individual packaging materials are higher compared to those of the collective recycling system of blue bins [14].

Finally, the tool calculates the impact of packaging waste via the use of the Eco-indicator scores, based on the standard 
indicators as defined in the Eco-indicator 99, Manual for Designers which refer to the Dutch conditions [15].

The database fields included in the TotalMillipointsTable are the following:

(i) Extraction: a numeric variable that contains the mPts associated with the extraction for each one of the packaging materials;

(ii) Processing: a numeric variable that contains the mPts associated with the processing for each one of the packaging materials;

(iii) Disposal: a numeric variable that contains the mPts associated with the final disposal for each one of the packaging materials;

(iv) Recycling: a numeric variable that contains the mPts associated with the recycling for each one of the packaging materials.

All relevant data are retrieved from the databases and manipulated using queries based on predefined criteria. Queries are also utilized for the execution of the calculations within a database or among the three aforementioned databases. More specifically, the products sum query calculates the sum of packaging materials associated with the consumer products by summing up the multiplication results of the actual quantities of products times the material(s) per product packaging. The results of the summation are appended to the TotalMaterialsTable.

3.2. Impact Reduction Algorithm. The program has also incorporated an impact reduction algorithm. Once the calculation of the total environmental impact has been performed, the user is prompt by a button reading "How to improve it?" Based on the initial product list of the user, the tool performs two checks based on a set of predefined logical criteria.

(i) At first, it checks the recycling database if there exists a better way of recycling for each packaging material. Thus, the total environmental impact is reduced via improved recycling.

(ii) Then, it checks the products database if there are similar products available in different packaging (e.g., refreshments offered in different packaging alternatives) that reduce the total impact by substituting their packaging material(s).

Based on those two checks, the tool prompts the user with the available alternatives for minimising the environmental impact of his/her product choices.

\section{Discussion}

The key advantage of the developed computer tool is its simplicity, both conceptual and operable. It can run on any personal computer running on a very popular operating system and having installed the affordable Microsoft Access software. On the other hand, this can also be a weakness of the tool, since the availability of a commercially available product is a prerequisite for the execution of the database. Based on the current version of the tool, the user can add products alongside their packaging to the database and update the recycling data per packaging method and material. Thus, the databases of the tool can be built up in order to include a vast variety of consumer products and on the other hand can stay always up to date following the improvements in the recycling infrastructure in Greece. Also, the conversion of the tool in an online platform will improve its visibility and usage by the consumers.

On the other hand, its main drawback is that the Ecoindicator 99 method and the subsequent estimation of environmental impact in mPts are based on Dutch data and, thus, in absolute terms, they are not representative of the packaging life cycle stages in Greece. However, this fact does not negate the usefulness of such a tool since the relative environmental impact assessment among product packaging waste and recycling practices remains intact.

The implications of the developed computer-aided tool for informing and raising consumer awareness on packaging waste recycling are enormous. Such a tool can be linked with the barcodes of supermarkets' and retailers' products. In such a case, the consumers will get a "packaging receipt" at the check-out cashiers which will inform them about the weight of every packaging material that they bought along with their goods.

\section{Conclusions}

A user-friendly computer-aided tool has been developed in Microsoft Access environment that aims at assisting the consumers to make environmentally friendly choices based on their consuming and recycling habits. The inputs to the tool are a list of consumer products and their quantities in addition to the recycling habits of the consumer. The tool, then, based on the available recycling rates per material and the predefined impact scale of Eco-indicator 99, calculates the total impact score of the initial list of products. The tool has also incorporated an impact improvement algorithm for offering educated alternatives to the user aiming at reducing the total environmental impact of his/her choices.

\section{Conflict of Interests}

The authors declare that there is no conflict of interests regarding the publication of this paper.

\section{References}

[1] K. Sonneveld, K. James, L. Fitzpatrick, and H. Lewis, "Sustainable packaging: how do we define and measure it?" 2005, http:// www.sustainablepack.org/database/files/spa\%20paper\%2022nd \%20iapri\%20symposium\%202005.pdf.

[2] G. Zhang and Z. Zao, "Green packaging management of logistics enterprises," Physics Procedia, vol. 24, pp. 900-905, 2012.

[3] J. Pasqualino, M. Meneses, and F. Castells, “The carbon footprint and energy consumption of beverage packaging selection and disposal," Journal of Food Engineering, vol. 103, no. 4, pp. 357$365,2011$. 
[4] R. Cahill, S. M. Grimes, and D. C. Wilson, "Extended producer responsibility for packaging wastes and WEEE: a comparison of implementation and the role of local authorities across Europe," Waste Management and Research, vol. 29, no. 5, pp. 455-479, 2010.

[5] ISO, "Environmental management-life cycle assessmentrequirements and guidelines," ISO 14040, International Organisation for Standardisation (ISO), Geneva, Switzerland, 2007.

[6] O. Ayalon, Y. Avnimelech, and M. Shechter, "Application of a comparative multidimensional life cycle analysis in solid waste management policy: the case of soft drink containers," Environmental Science and Policy, vol. 3, no. 2-3, pp. 135-144, 2000.

[7] M. P. Hekkert, L. A. J. Joosten, E. Worrell, and W. C. Turkenburg, "Reduction of $\mathrm{CO}_{2}$ emissions by improved management of material and product use: the case of primary packaging," Resources, Conservation and Recycling, vol. 29, no. 1-2, pp. 3364, 2000.

[8] M. B. Hocking, "Paper versus polystyrene: a complex choice," Science, vol. 251, no. 4993, pp. 504-505, 1991.

[9] "The ecoinvent database," 2013, http://www.ecoinvent.org/database/.

[10] L. Quinn and A. J. Sinclair, "Policy challenges to implementing extended producer responsibility for packaging," Canadian Public Administration, vol. 49, no. 1, pp. 60-79, 2006.

[11] V. S. Rotter, "Waste management and producer responsibility: a score behind-a new ahead," Waste Management and Research, vol. 29, no. 9, pp. 889-890, 2011.

[12] Hellenic Recycling Agency, 2012, http://www.eoan.gr/.

[13] P. Nimse, A. Vijayan, A. Kumar, and C. Varadarajan, "A review of green product databases," Environmental Progress, vol. 26, no. 2, pp. 131-137, 2007.

[14] K. Abeliotis, K. Karaiskou, A. Togia, and K. Lasaridi, "Decision support systems in solid waste management: a case study at the national and local level in Greece," Global Nest Journal, vol. 11, no. 2, pp. 117-126, 2009.

[15] Pré Consultants, "Ecoindicator99-Manual for designers," 2012, http://www.pre-sustainability.com/download/manuals/EI99_ Manual.pdf. 

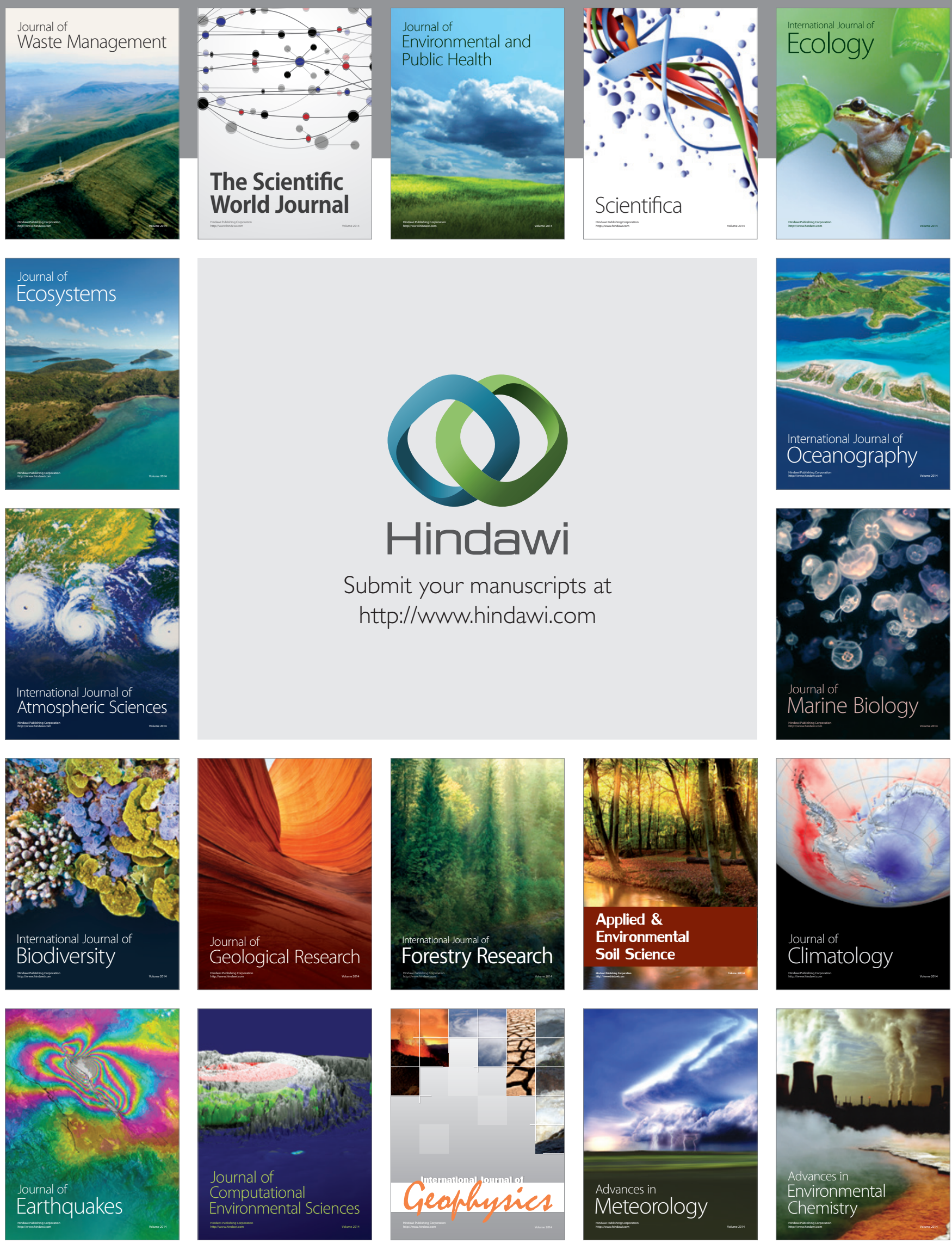